Trans

continentales
Transcontinentales

Sociétés, idéologies, système mondial

$8 / 9 \mid 2010$

Des migrations aux circulations transnationales

\title{
Un itinéraire de recherche
}

L'étude de la mondialisation par le bas

Entretien mené par Boris Petric

Alain Tarrius

\section{(2) OpenEdition}

12 Journals

Édition électronique

URL : http://journals.openedition.org/transcontinentales/1301

DOI : 10.4000/transcontinentales. 1301

ISBN : 978-2-7351-1557-0

ISSN : $1775-397 X$

Éditeur

Editions de la maison des sciences de l'homme

Édition imprimée

Date de publication : 31 décembre 2010

ISSN : 1950-1684

\section{Référence électronique}

Alain Tarrius, «Un itinéraire de recherche », Transcontinentales [En ligne], 8/9 | 2010, document 17, mis en ligne le 07 décembre 2011, consulté le 25 septembre 2020. URL : http://journals.openedition.org/ transcontinentales/1301; DOI : https://doi.org/10.4000/transcontinentales.1301

Ce document a été généré automatiquement le 25 septembre 2020.

Tous droits réservés 


\title{
Un itinéraire de recherche
}

\author{
L'étude de la mondialisation par le bas \\ Entretien mené par Boris Petric
}

\section{Alain Tarrius}

1 Transcontinentales : Avant d'être professeur de sociologie à Toulouse, comment avez-vous commencé vos recherches sur les migrations?

Alain Tarrius : J'ai été tout d'abord chercheur à l'Institut national des transports et de la sécurité (INRETS). Pour moi, cette première expérience professionnelle a été très intéressante pour étudier des questions de mobilité. Cet institut rassemble majoritairement des ingénieurs qui réfléchissent à l'organisation des flux. J'étais en quelque sorte le « sociologue de service » qui devait penser l'articulation entre l'espace et le tissu social. Cette période a été pour moi très féconde car j'ai eu beaucoup de liberté pour faire des enquêtes passionnantes.

Plus tard, j'ai été sollicité par Gaston Defferre. Le maire de Marseille m'a fait part d'un problème, dans un quartier de sa ville, concernant les activités commerciales de Taxiteurs dans le quartier de Belsunce. Je suis allé voir de plus près et j'ai entrevu une caverne d'Ali Baba. J'ai découvert un quartier où se déroulait sous mes yeux un vaste commerce international assez informel. Il y avait alors un contraste très fort entre l'état dévasté de ce quartier du centre ville de Marseille et son dynamisme commercial. En fait, Belsunce était un centre économique fantastique que les pouvoirs politiques marseillais sous-estimaient. Plus de 700000 personnes circulaient et généraient un chiffre d'affaire évalué plus tard par la Caisse des dépôts et consignation à plus de 3 milliards de francs. Face à la découverte d'un monde économique et social souterrain, je suis allé vers des paradigmes phénoménologique et interactionniste, j'ai privilégié l'observation participante pour pénétrer cette réalité sociale. C'était un phénomène qui restait dans l'ombre quand j'ai commencé à l'étudier, il n'y avait pas de chiffres et de statistiques. J'ai donc mené des enquêtes qualitatives auprès de tous ces marchands (1986-87). J'ai essayé, à ce moment-là, de partir vers la théorisation d'un paradigme autour de la mobilité en essayant de comprendre l'articulation de différents espaces local, régional, national et lointain - allant aussi bien vers le Maghreb que vers la Turquie. 
4 En 1991, les réseaux d'échanges licites ont pris un essor encore plus grand. Cela allait des petites marchandises comme des téléviseurs, des antennes paraboliques, des machines à laver, mais il y avait également des tracteurs, des moissonneuses batteuses qui étaient revendus et expédiés vers le Maghreb. Cette même logique s'observe aussi pour les pays de l'Est. J'ai commencé alors à réfléchir au problème de l'investissement productif car la théorie dominante reposait sur la question de la main d'œuvre. On pourrait dire qu'idéologiquement j'étais très isolé et que j'ai eu du mal à convaincre mes collègues de prendre au sérieux la question de la circulation pour comprendre aussi bien les évolutions de notre économie que de notre société en général.

5 Avec l'ouverture à l'Est, les choses se sont encore amplifiées, ce qui a donné lieu au développement d'un commerce très intense entre des villes ouest européennes et les pays de l'Est. C'est le moment où la migration s'est aussi intensifiée dans certains pays méditerranéens. Entre 1991 et 2001, il y a eu plus de trois millions de Marocains qui sont partis dans une aventure migratoire.

6 Dès 1991, j'ai été attentif à ces Marocains, rejoints rapidement par les Sénégalais. J’ai alors proposé la notion de territoire circulatoire en opposition à celle d'espace pour rendre compte de ce phénomène. La notion de territoire vient souligner l'intensité des relations sociales et économiques qui se construisent dans les déplacements. La récurrence des rapports donne des dimensions de continuum à ces territoires, des étapes qui les bornent aussi d'une certaine manière.

7 En 1995, j'ai publié un ouvrage (Arabes de France dans l'économie souterraine mondiale, Paris, l'Aube) qui a changé le regard de ma communauté sur mon travail. L'accueil scientifique est devenu chaleureux de la part de certains de mes collègues ce qui m'a permis de partir dans une nouvelle dynamique de recherche.

8 En 2002, j'ai publié un ouvrage intitulé la mondialisation par le bas faisant référence à l'article d'un chercheur américain Alejandro Portes qui s'intéressait au caractère transnational des phénomènes migratoires aux États-Unis. J'ai sorti un ouvrage intitulé les nouveaux cosmopolitismes qui touchait à la question de la mobilité et de l'intégration.

TC. : En 1991, c'est aussi de nouvelles relations avec les pays de l'Est. Constatezvous, à ce moment-là, le croisement de ces flux venant du sud et de l'est et traversant l'Europe occidentale?

10 A.T. : À partir de la fin des années 1990, j'ai commencé à regarder aussi les flux migratoires venus de l'est de la Méditerranée. Dans cette perspective, j'essaye de travailler encore sur cette réflexion majeure de la migration circulatoire pour essayer de trouver un concept qui rende compte de ces pratiques de transmigrants, c'est-à-dire de personnes qui sont dans des logiques d'étapes qu'elles viennent du Maroc, d'Algérie, de Pologne ou d'Afghanistan. Ces individus construisent des trajectoires inouïes dont notre société dominante soupçonne finalement peu l'existence. Dans une conception classique de la société, nous ne croisons pas ces gens-là, mais il existe pourtant tout un ensemble de personnes qui vivent en réseau et qui circulent et font circuler des marchandises, des personnes et des pratiques sociales qui contribuent à modifier notre société et les leurs.

11 On pense, par exemple, qu'il serait plus de 5000 transmigrants à circuler ainsi avec des relais, des étapes de la Bulgarie à la France et l'Espagne. Je pourrais vous parler du phénomène de ce que l'on appelle les médecins égyptiens. Ce sont des médecins diplômés venant de pays arabes qui font des consultations nomades auprès des 
populations musulmanes en Europe occidentale. Ils savent faire des diagnostics qui respectent certains de leurs préceptes, notamment dans le rapport au corps. Ils ne dénudent pas le corps des femmes et élaborent un diagnostic grâce un savoir différent de la médecine actuelle (prises des pouls, observation de l'iris, des oreilles). Ce n'est pas un rejet catégorique de la médecine telle qu'elle est pratiquée en France. D'ailleurs, en fonction de la gravité, ils peuvent envoyer les gens aux urgences. Cependant, ils peuvent faire aussi, dans certains cas, leur propre diagnostic et proposer des stratégies thérapeutiques. Ils s'appuient alors sur des jeunes qui maîtrisent Internet et qui peuvent commander sur des sites Internet toute sorte de médicaments.

Ce genre de pratiques révèle typiquement des territoires circulatoires dont je parle. Ils sont très nombreux dans les grandes villes françaises et ouest européennes. Ce sont les mêmes chemins que prennent les commerçants. Ce sont aussi les chemins des femmes venant de l'Est qui se prostituent. Il faut savoir qu'il y a plus de 270 bordels sur le front de l'Est espagnol. Ces femmes restent un temps dans un pays puis peuvent poursuivre leur chemin. Dans ces trajectoires, elles considèrent l'Espagne comme une étape et essayent de rentrer dans une carrière internationale en considérant que l'objectif est de parvenir à travailler en Allemagne ou en Hollande avant de retourner chez elles en Moldavie, en Albanie (Europe de l'Est et Ex-URSS).

13 Évidemment, les rythmes ne sont pas les mêmes en fonction des territoires circulatoires, mais il y a ces logiques d'étapes que l'on retrouve. Il y a les femmes, les commerçants, les médecins.

14 J'ai aussi beaucoup travaillé sur ce que l'on appelle les Afghans qui sont en fait souvent des Baloutches qui font transiter tout un ensemble de produits électroniques des Émirats Arabes Unis vers l'Europe. C'est ce que l'appelle le poor to poor permettant d'ouvrir un marché considérable aux firmes du Sud-Est asiatique. Il y a ici un paradoxe très important : les transmigrants vivent leur trajectoire comme une aventure. Ils ont l'impression d'être relativement libres dans la construction de leur trajectoire de vie. Eh bien, je dirais qu'ils sont l'objet d'une logique économique qui leur échappe totalement.

15 Leur compétence est à vendre, c'est le franchissement des frontières qui renvoient à la question de la corruption et donc, aussi, à la relation avec nos bureaucraties.

\section{TC. : Pouvez-vous revenir sur les questions méthodologiques et la difficulté de} travailler sur un monde largement souterrain?

17 A.T. : J'ai vécu l'expérience de circulation de ces transmigrants pendant plusieurs mois en suivant plusieurs d'entre eux. Ce travail ne peut se réaliser qu'en construisant un lien fort avec les personnes que l'on étudie. Au départ, pour me justifier auprès d'eux, j'expliquais que je réfléchissais à des aspects logistiques pour une grande société de transports. Cette histoire ne tenait pas. Alors, le terrain vous prend et vous donne un statut permettant de construire un lien. Ma place parmi eux s'est construite peu à peu à leur contact au point, qu'à un moment donné, les choses ne dépendaient pas uniquement de ce que je pouvais dire. Pour être crédible et être accepté dans cet univers, des transmigrants m'ont inventé et imposé un prétexte pour légitimer ma présence parmi eux. J'étais, par exemple le père d'un Français qui se serait battu avec eux au Kosovo. La condition pour faire ce type de travaux est d'accompagner. Il y a des sociabilités originales qui se construisent dans la mobilité. Ces transmigrants 
acquièrent des compétences et il faut à l'évidence passer du temps au cœur de ce processus pour comprendre cette inventivité sociale.

Je dirais que lorsque nous sommes déjà dans une démarche statistique, le phénomène est déjà mort. Si on peut les compter, cela signifie que le système ne fonctionne plus.

J'ai passé beaucoup de temps dans les ports à compter les gens descendants et montants dans les bateaux, à observer les douanes. Je parle une sorte de broken english, ce que parlent tous les migrants que j'ai côtoyé. Quelqu'un qui parlerait bien anglais serait d'ailleurs immédiatement suspect.

C'est une capacité à aller vers les gens, créer un lien. En réalité, les événements sont mineurs au quotidien. Mon travail consiste à vérifier in situ des interactions, des régularités, des situations qui se rattachent à nos problématiques et à nos populations. Cela demande une présence assez longue sur le terrain.

21 D'un point de vue méthodologique, ces circulations donnent une image très mouvante de la ville qui se recompose sans arrêt en fonction de ces échanges. C'est très important de prendre en compte cette temporalité pour construire sa méthodologie.

TC.: Vos recherches vous donne une perspective intéressante pour penser les politiques publiques. Quel regard portez-vous sur le rôle de l'État?

23 A.T. : Je dirais que les schémas d'intégration classiques sont obsolètes. Plus je travaille sur ces transmigrants et plus je pense l'hypothèse que les étapes qu'ils font, en Italie, en France, en Espagne, sont dans l'altérité, dans le contact. On peut d'ores et déjà signaler des trajectoires d'intégration européennes. Un certain nombre de compétences fondées sur la mobilité peut être une forme d'intégration à l'espace européen. Ils deviennent des marchands internationaux avec une forme de compagnonnage européen.

Pour comprendre les mutations actuelles, je reviendrais à la sagesse de Park pensant l'évolution de la ville de Chicago. Ce dernier dit le développement de la ville. Il faut certes comprendre les investissements fonciers, l'économie, mais il faut aussi comprendre ces lieux de la nuit où se rencontrent des mondes différents où se renouvèlent des nouveaux modes de sociabilité. Je crois que cette problématique est exportable à des zones entières qui ont comme caractéristique de favoriser des brassages entre les échanges de produits licites ou illicites. Les gens fonctionnent sur des logiques multiples, sur des appartenances multiples. Il y a actuellement des zones où de multiples échanges se réalisent. Ces espaces, je les ai trouvés sur l'ancienne frontière entre l'Est et l'Ouest, sur la Mer noire entre la Turquie et la Géorgie. Les rives de la Mer noire sont typiquement des lieux où s'élabore une nouvelle réalité sociale sur un mode complémentaire comme dans la ville de Trabzon. Ces milieux de la transgression, comme le commerce d'héroïne par exemple nous rattache beaucoup plus qu'on ne le croit au fonctionnement légal et normatif de notre société.

25 Prenez, par exemple, le cas de la corruption : elle touche à une question plus générale, celle de la relation avec la bureaucratie. On peut observer tout un ensemble d'échanges qui se négocient. Le capitaine de douanes vous propose des faveurs mais vous pouvez allez voir aussi le capitaine de police. J'ai acheté par exemple un passeport français 700 euros à Burgas avec un vrai numéro. Cela existe aussi dans le sud de l'Italie vers Bari et aussi dans le sud de l'Espagne en Andalousie vers Tarifa et Malaga. Ces zones ont la caractéristique de fédérer plusieurs frontières.

TC. : Dans vos recherches de terrain, en quoi les instituts français de recherche à l'étranger ont-ils été un élément important? 
27 A.T. : J'ai connu tout d'abord Alain Roussillon qui dirigeait le centre Jacques Berque à Rabat, puis le CEDEJ au Caire. Beaucoup de mes collègues de MIGRINTER ont été en poste dans ces instituts à Beyrouth, à Damas ou à Istanbul. J'ai immédiatement bénéficié dans mes différents travaux de ce magnifique réseau des instituts français. Plus tard, de nombreux projets de recherche ont été initiés sur la thématique des nouvelles caravanes commerciales. Et je me suis moi-même appuyé sur ces instituts lorsque j'ai travaillé au Maroc, en Turquie.

À Istanbul, par exemple, j'ai reçu une aide très précieuse de Jean-François Pérouse qui s'occupe de l'observatoire urbain à l'IFEA. Je peux dire que ces instituts m'ont permis d'aller beaucoup plus vite. Des chercheurs m'ont fait bénéficié de leur contacts, ils m'ont aidé en me signalant les lieux où je pouvais construire mes terrains. Et je dirais que ces instituts ont été un précieux atout pour la construction de mes recherches dans ces différents pays dont je n'étais pas forcément familier.

\section{AUTEUR}

\section{ALAIN TARRIUS}

Professeur à l'Université de Toulouse-le-Mirail, membre des laboratoires CNRS : LISSTCERS Toulouse et Migrinter Poitiers. 